\title{
Fuselage Damage Locator System
}

\author{
Yair Wiseman ${ }^{1}$, \\ ${ }^{1}$ Computer Science Department \\ Ramat-Gan 52900, Israel \\ wiseman@cs.biu.ac.il
}

\begin{abstract}
Damaged fuselage can cause a fatal catastrophe that will claim numerous lives. This paper suggests the use of a regular digital camera that can generate JPEG images so as to locate the damaged part of the fuselage. The digital camera takes pictures of the fuselage. The signal processing method of JPEG assumes that the taken image is to a certain extent smooth. As a result when there is a sharp change in a particular block of an image, the value of many of its frequency coefficients will be high and it will be compressed into many more bits; therefore, if the image is overly large, an embedded computer system can track down the damage. In that case, sometimes an action can be taken so as to reduce the devastative effects of the damage.
\end{abstract}

Keywords: Fuselage, Discrete Cosine Transform, JPEG

\section{Introduction}

At April 1, 2011, a Boeing 737-3H4 flew from Phoenix, Arizona to Sacramento, California. Near Yuma, Arizona, a mysterious sound was recorded on the cockpit area microphone. Few seconds later, the captain noticed that the airplane had rapidly lost cabin pressurization and called for oxygen masks on. Steady sounds with increased wind noise were heard on the cockpit voice recording. The captain announced an emergency with air traffic control. The cabin crew began to search the source of the noises and found a 2-foot hole in the fuselage. The aircraft was diverted to Yuma International Airport. The airplane landed there without further incident. The damaged part of the fuselage can be seen at Figure 1. The cabin crew in this case eventually indeed succeeded to find the damage part; however it took some time to find the damaged part and in such cases time is very crucial [1].

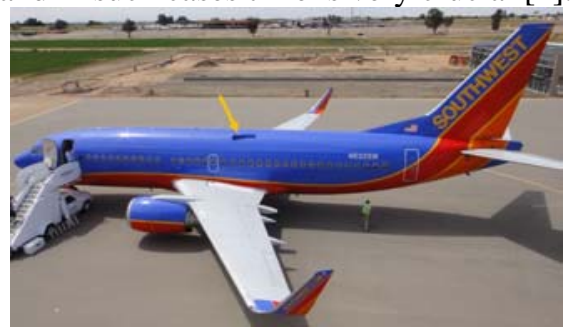

Fig. 1. Dangerous fuselage damage 
Advanced Science and Technology Letters

Vol.37 (Signal Processing 2013)

We suggest using an ordinary digital camera to locate the damaged part. Nearly all digital cameras can produce JPEG pictures. JPEG images have many advantages like the ability of being decoded in parallel [2], the straightforwardness of adaptation for new compression methods $[3,4]$ and the capability of flexible implementation for hardware from different vendors [5].

Images are often stored in a compressed standard. A naive approach for image processing on compressed images would be to decompress the image and then running the image processing algorithm on the original image data. Instead, for some image operations, we can act on the compressed data directly. This gives us two benefits: first, we can use the standard digital cameras without a need to adjust the digital camera; second, the signal processing used by JPEG can let us use the frequency information embedded in the compressed data.

\section{Fuselage Damage Locator System Using JPEG}

JPEG is a well known standardized image compression technique. JPEG loses information, so the decompressed picture is not the same as the original one. By adjusting the compression parameters, the degree of loss can be adjusted. The wide use of JPEG is because of two fundamental reasons: storing full color information and reducing the size of image files, so as to avoid high traffic on the network and avoiding memory pressure [6]. JPEG is an eminent format and is described in many places e.g. [7,8]. The JPEG standard is based on the DCT paradigm [9]. The DCT changes the picture into frequency space. The frequency coefficients, which are very low magnitude, are rounded to zero. When most of the coefficients in a block are zero or very low magnitude: The compression algorithm will give a very short bits sequence for such a block. Zero sequences are treated very efficiently by JPEG compression and the results will be only few bytes.

When there is a drastic change in a block of $8 \times 8$, the value of many frequency coefficients will be high. Such a sequence will be compressed into many more bits. JPEG's standard stipulates that the block's size will be $8 X 8$ pixels, but the algorithm will be obviously good for other small NXN pixels size too.

When looking for the contour of an object, the goal will be to locate the object's border. The idea is to break the compressed file into its original blocks, then look in the compressed file for long bit sequences. The blocks which are compressed into long bit sequences, are presumed to be the object's border. In our implementation we took a simpler approach. We take pictures of the fuselage. We actually take a close picture of each part of the fuselage. If the entire size of a picture is above a certain threshold, we will consider this part of the fuselage as a damaged part.

If we have no idea what the threshold value should be, we can examine the probability density function (PDF) of the block representation to select a suitable value. In the uncomplicated case the PDF should be mono-modal and we set the value in the inflection point $[10,11]$. The JPEG file reports the difference of magnitude between the DC's coefficients of a previous block relative to the current block. In the case of a white or black area there are no changes in the coefficients' magnitudes. This type of block is encoded as six bits by the JPEG standard: 0,0,1,0,1,0. The " 00 " 
reflects that there are no differences between the values of the previous and the current block's DC coefficients, and "1010" symbolizes the end of the block. If there is a difference between the intensity of the DC coefficients of the previous and the current blocks, the size of the encoding block will be slightly larger. For example, a block which encodes a sharp change from white to black is represented by a wide range of frequency coefficients. It is easy to select a threshold that delimits the edges of the shape from the rest of the image $[12,13,14]$.

\section{Experiments}

We examined our technique on some damaged fuselages and tried to check whether we succeed to locate the damaged part. We used an Olympus FE-170 digital camera with resolution of 2112 X2816. Many of the fuselage damages are clear. It appears that many wildlife strike aircrafts [15] and in such cases the damage is usually quite large. Since the establishment of the Federal Aviation Administration's (FAA) National Wildlife Strike Database at 1990, 99,411 reported wildlife strikes to airplanes have been reported. The result of these strikes causes more than 200 human lives lost worldwide as well as financial losses (direct and indirect) of at least \$1.2 billion annually to civil aviation worldwide. Particularly, more than $\$ 625$ million of financial losses annually just in the United States [16].

However, sometimes there are smaller damages. One major reason for smaller damages is hail [17]. At July 14, 2011, More than 100 flights have been called off at Denver International Airport after hail damaged about 40 aircrafts and stranded about 1,000 passengers overnight. The damages were usually not large as can been seen in Figure 2.

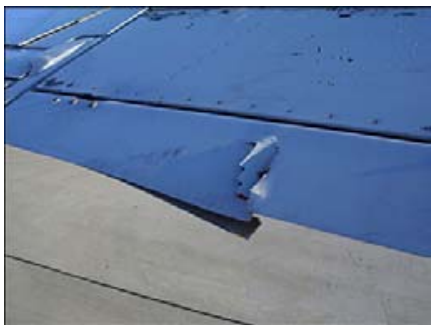

Fig 2. Hail Damage in Denver International Airport

As a matter of fact, the use of our system for large and clear damages is less important, although sometimes a cabin crew member under stress can need a help even such cases; however when a small and indistinguishable damage can make lower the pressure in the cabin and locating it without delay can be an imperative.

\section{Conclusions}

Fuselage is very risky and can cause too many loss of life. Automatic inspection of aircraft fuselage is critical and vital as was noted in [18]. The suggested system in this 
Advanced Science and Technology Letters

Vol.37 (Signal Processing 2013)

paper can be mounted in an aircraft and it may save lives. In this paper, we explained how a system using simple equipment - digital camera can detect damages in a fuselage.

\section{References}

1. Dalton, R. P., Cawley, P. and Lowe, M. J. S., The potential of guided waves for monitoring large areas of metallic aircraft fuselage structure. Journal of Nondestructive Evaluation, Vol. 20(1), pp. 29-46, 2001.

2. Klein S. T. and Wiseman Y., Parallel Huffman Decoding with Applications to JPEG Files, The Computer Journal, Oxford University Press, Swindon, UK, Vol. 46(5), pp. 487-497, 2003.

3. Wiseman Y., Burrows-Wheeler Based JPEG, Data Science Journal, Vol. 6, pp. 19-27, 2007.

4. Klein S. T. and Wiseman Y., Parallel Lempel Ziv Coding, Journal of Discrete Applied Mathematics, Vol. 146(2), pp. 180-191, 2005.

5. Wiseman Y., A Pipeline Chip for Quasi Arithmetic Coding, IEICE Journal - Trans. Fundamentals, Tokyo, Japan, Vol. E84-A No.4, pp. 1034-1041, 2001.

6. Reuven, M., and Wiseman, Y., Medium-term scheduler as a solution for the thrashing effect. The Computer Journal, 49(3), 297-309, 2006.

7. Information Technology - Digital Compression and Coding of Continuous-Tone Still Images Requirements and Guidelines - International Standard ISO/IEC 10918-1, 1993.

8. Wallace, G. K., The JPEG Still Picture Compression Standard Communication of the ACM 34, pp. 3-44, 1991.

9. Britanak, V. and Yip P. C., and Rao K. R.. Discrete cosine and sine transforms: general properties, fast algorithms and integer approximations, Elsevier, 2010.

10. Wiseman Y. and Fredj E., Contour Extraction of Compressed JPEG Images, ACM - Journal of Graphic Tools, Vol. 6 No. 3, pp. 37-43, 2001.

11. Fredj E. and Wiseman Y., An O(n) Algorithm for Edge Detection in Photos Compressed by JPEG Format, Proc. IASTED International Conference on Signal and Image Processing SIP2001, Honolulu, Hawaii, pp. 304-308, 2001.

12. Y. Wiseman, "Take a Picture of Your Tire!", Proc. IEEE Conference on Vehicular Electronics and Safety (IEEE ICVES-2010) Qingdao, ShanDong, China, pp. 151-156, 2010.

13. Y. Wiseman, The Effectiveness of JPEG Images Produced By a Standard Digital Camera to Detect Damaged Tyres, World Review of Intermodal Transportation Research, Vol. 4(1), pp. 23-36, 2013.

14 . Y. Wiseman, Camera That Takes Pictures of Aircraft and Ground Vehicle Tires Can Save Lives, Journal of Electronic Imaging, Vol. 22(4), 041104, 2013.

15. Blackwell, B. F. DeVault, T. L. Fernández-Juricic, E. and Dolbeer, R. A., Wildlife collisions with aircraft: a missing component of land-use planning for airports, Landscape and Urban Planning, 93(1), 1-9, 2009.

16. Martin, J. A., Belant, J. L., Devault, T. L., Blackwell, B. F., Burger Jr, L. W., Riffell, S. K. and Wang, G., Wildlife risk to aviation: a multi-scale issue requires a multi-scale solution. Human-Wildlife Interactions, 5(2), 198-203, 2011.

17 Souter, R. K., and Emerson, J. B. Summary of available hail literature and the effect of hail on aircraft in flight (No. NACA-TN-2734). National Aeronautics and Space Administration Washington DC, 1952.

18. Alan J. Stolzer, Carl D. Halford and John J. Goglia, Safety Management Systems in Aviation, Chapter 3 - Principles of Quality Management, MPG Books Group, UK, 2010. 